What Do Cows Drink? A Systems Factorial Technology Account of Processing Architecture in Memory Intersection Problems

\author{
Zachary L. Howard ${ }^{1}$, Bianca Belevski ${ }^{1}$, Ami Eidels ${ }^{1}$ and Simon Dennis ${ }^{1,2}$ \\ ${ }^{1}$ School of Psychology, University of Newcastle, Australia \\ ${ }^{2}$ School of Psychology, Melbourne University, Austalia
}

\begin{abstract}
Author Note
Correspondence concerning this article may be addressed to: Zach Howard, School of Psychology, University of Newcastle, Callaghan NSW 2308, Australia; Email: zachary.howard@uon. edu. au
\end{abstract}

The authors have no competing interests to declare. This research was supported by an Australian Government Research Training Program (RTP) Scholarship awarded to Zachary Howard. 


\begin{abstract}
It has long been known that cues can be used to improve performance on memory recall tasks. There is evidence to suggest additional cues provide further benefit, presumably by narrowing the search space. Problems that require integration of two or more cues, alternately referred to as memory intersections or multiply constrained memory problems, could be approached using several strategies, namely serial or parallel consideration of cues. The type of strategy implicated is essential information for the development of theories of memory, yet evidence to date has been inconclusive. Using a novel application of the powerful Systems Factorial Technology (Townsend \& Nozawa, 1995) we find strong evidence that participants use two cues in parallel in free recall tasks - a finding that contradicts two recent publications in this area. We then provide evidence from a related recognition task showing that while most participants also use a parallel strategy in that paradigm, a reliable subset of participants used a serial strategy. Our findings suggest a theoretically meaningful distinction between participants strategies in recall and recognition based intersection memory tasks, and also highlight the importance of tightly controlled methodological and analytic frameworks to overcome issues of serial/parallel model mimicry.
\end{abstract}

Keywords: memory, systems factorial technology, sft, serial processing, parallel processing, intersection 


\section{What Do Cows Drink? A Systems Factorial Technology Account of Processing Architecture in Memory Intersection Problems}

\section{Introduction}

We often attempt to recall a name of a book, or movie, given cue(s) providing partial information. If we recall the movie was "with Robin Williams" - the number of candidate movies is large and we would probably struggle to convey to others which movie it was. But if you were given the prompt that the main character was "a man dressed as a woman", you may immediately identify the movie "Mrs Doubtfire". We solve problems like this everyday with little thought. For example, when selecting the fastest route home accounting for distance and traffic, or planning dinner based on the available ingredients, we are integrating several constraints in order to retrieve a remembered route or recipe. A more salient example is solving a crossword, which explicitly requires retrieval of solutions that meet both meaning and form constraints. Psychologists have implicated such "multiply constrained optimization" in concepts as varied as recall memory (Craik, 2002), forming impressions of strangers (Kunda \& Thagard, 1996), sentence parsing (Taraban \& McClelland, 1990), and judicial decision making (Robbennolt, MacCoun, \& Darley, 2010).

Throughout this manuscript, we term the solution to such 'constrained' memory problems the "intersection" of multiple cues. An intersection arises when two (or more) cues together probe the same memory. Note that although in the above example the cues may well have been seen together previously, two cues may have an intersection even if they are unrelated (consider that cows are rarely seen drinking milk yet most of us have fallen for the trick question nonetheless). Also important is that while retrieving intersections is often helpful (e.g., to quickly recall the best route home, given the current traffic, when we learn of an accident on our primary route), sometimes they can hinder (as cows drink water, not milk). In this way, much like visual illusions, the ubiquity of the processes are evidenced by the relatively few cases in which they fail us. The primary question of this study is whether multiple constraints are considered in parallel or in serial, 
when identifying an intersection of multiple cues. For illustration, in the Venn diagram in Figure 1, "Mrs Doubtfire" lies at the intersection of the two clues provided. A parallel strategy implies operating on each cue simultaneously, which should direct the process to the area of overlap. A parallel search is generally consistent with the way some intersections seem to 'pop out', such as when asked what cows drink (see e.g., Treisman \& Gelade, 1980). A serial process would instead consider cues one-at-a-time. A serial process has been implicated by several authors in recent years (Smith, Huber, \& Vul, 2013; Davelaar, 2015), yet serial processes are known to be generally less efficient than parallel searches (Townsend \& Nozawa, 1997).

Serial and parallel processes have been shown to be difficult to disentangle (Townsend, 1972), necessitating specialized analysis techniques that were not used in previous studies. To re-examine the question of serial vs parallel processing in memory intersections we extended the powerful Systems Factorial Analyses framework (SFT; Townsend \& Nozawa, 1995) to the novel domain of semantic memory. Next, we provide a formal introduction of the paradigm of constrained memory search, and then detail a series of studies that show most participants solve intersection problems using a parallel process across both recall and recognition memory paradigms. 


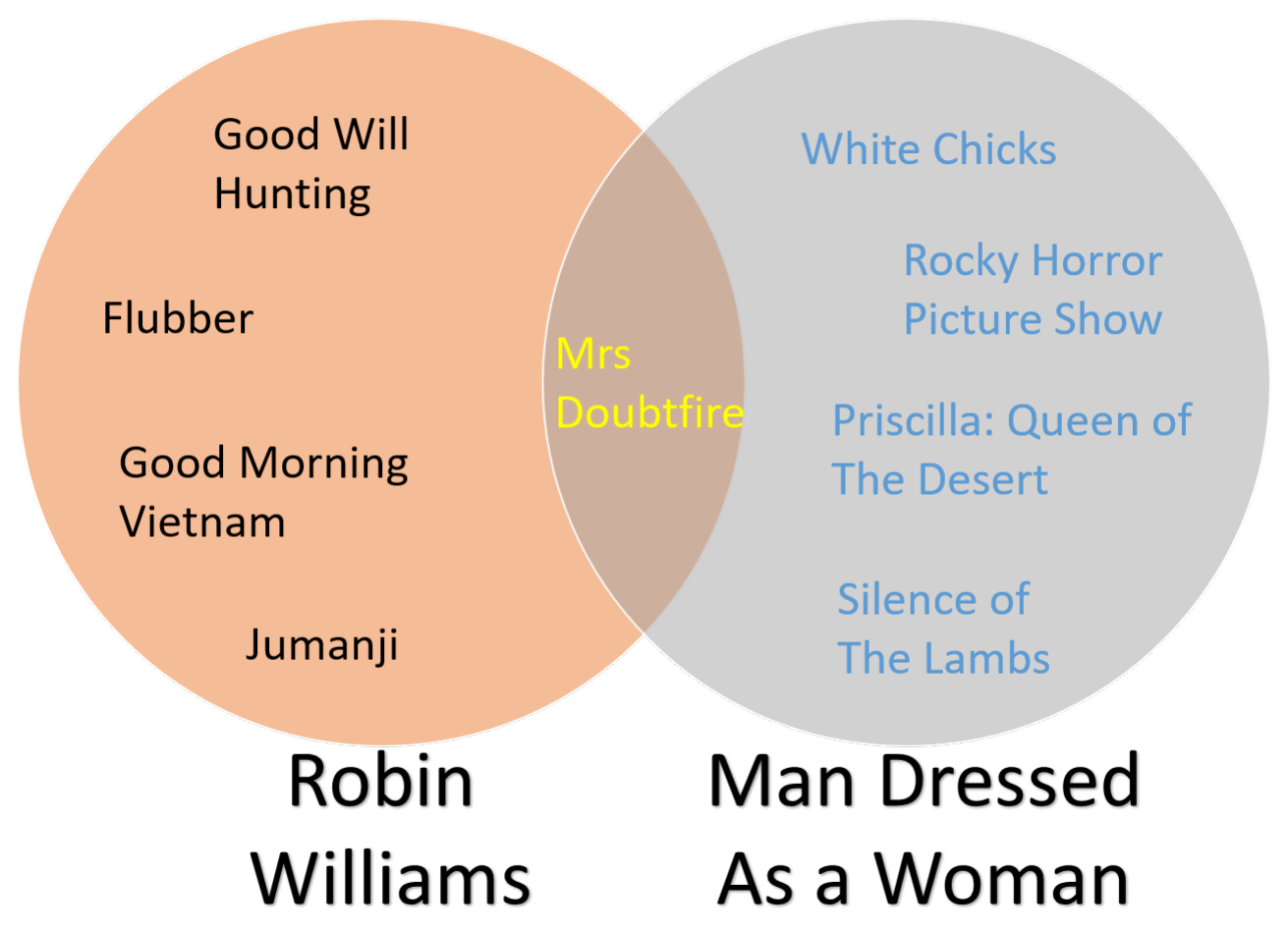

Figure 1. Venn Diagram highlighting the intersection point of two memory cues. Each cue prompts towards a number of items in memory, but only the intersection (in yellow) is prompted by both cues.

\section{Constrained Memory Search}

Attempting to recall something with no guidance is an example of a recall memory task (Britannica, 1965, entry "recall"). Although recalling information from memory is critical to daily functioning, unconstrained memory searches are difficult, and often we cannot remember something even with great effort. Many studies have shown that providing a 'cue' can greatly improve recall memory (Tulving \& Pearlstone, 1966; Thomson \& Tulving, 1970; Tulving \& Osler, 1968; Tulving \& Thomson, 1973; Watkins \& Tulving, 1975), and there is ample evidence that additional cues further improve memory retrieval. For example, Rubin and Wallace (1989) demonstrated how words that are unlikely to be freely generated with a single cue are almost certain to be generated given the provision of two intersecting cues (e.g., "a mythical being" and "rhymes with toast" almost always 
elicited the target response "ghost" when presented together, but not when presented separately). Rubin and Wallace demonstrated that "the observed effect of dual cuing greatly exceeds what usually would be expected from the independent strengths of the single cues" (Rubin \& Wallace, 1989, p. 703). This is strong evidence that participants were able to combine the constraints provided by each cue in order to draw from the intersection space instead of simply using either cue independently. Frey and Adesman (1976) showed that experienced chess players remembered the location of pieces better when they were constrained by both the rules of chess and a logical sequence of moves than either of those constraints in isolation. Participants in Massaro, Weldon, and Kitzis's (1991) study identified words better when they were given both orthographic and semantic prompts then either alone (and, as with Rubin and Wallace's study these cues were used multiplicatively).

Multiple cues, when available, could be used in sequence or simultaneously. Thus, the results of Rubin and Wallace (1989) and other related studies could arise from either a parallel or serial strategy. There are several examples of dual-memory cue scenarios in which sequential (serial) treatment of cues seems less likely. For example, both Rubin (1995) and Neisser (2014) have proposed the dual constraints of memory and rhyme as a reason for the successful transmission of oral traditions, epic poetry, and even children's rhymes such as eenie-meenie-miney-mo. The real-time constraints of meaning, meter, rhyme etc. so tightly constrain the songs that the words naturally come to the singer. This 'on-the-fly' constraint suggests a parallel process is involved, or else the singer might select a word that rhymes but does not fit the story. Notably though, serial and parallel processes can mimic each other, and data without temporal information is insufficient to distinguish between these processes (Townsend, 1972; Howard, Eidels, Silbert, \& Little, 2017). Several approaches have been offered to overcome mimicry issues (Townsend, 1990), including SFT (Townsend \& Nozawa, 1995; Little, Altieri, Fific, \& Yang, 2017; Altieri, Fifić, Little, \& Yang, 2017). We combine SFT with a variant of the remote associates task in the current 
study.

\section{Remote Associates Task}

One of the most notable experimental examples of 'multiply constrained' problem solving research is the Remote Associates Task (RAT; Mednick, 1968). In the RAT, three 'cue' words are provided (e.g., "moon", "comb" and "bee") and participants are asked to identify a target word that is connected to all of them ("honey"). Many theoretical accounts of the RAT assume a parallel, spreading activation account in which each cue is activated, then triggers associated nodes that become activated as well (e.g. Bolte, Goschke, \& Kuhl, 2003; Topolinski \& Strack, 2008). The target should be 'activated' multiple times, and would thus be more likely to trigger a response. Without such parallel activation a participant would seemingly need to draw 'associates' from any of the three cues, then compare that candidate to the others. Two recent considerations of the RAT conclude that participants do exactly that - generating candidate responses corresponding to only one of the cues at a time (Smith et al., 2013; Davelaar, 2015).

Smith et al. (2013) modified the RAT such that while trying to solve the problem (which can take up to 2 minutes), participants would type in any words they considered might be a solution. The authors argued that this adaptation allowed "online" investigation of the underlying search process, compared with previous approaches that were limited to investigating only the final outcome (Smith et al., 2013, p .65). The results of this study (which were subsequently replicated by Davelaar, 2015 using a similar design) suggested that participants were drawing candidate responses from one cue at a time. A typical series of responses showed several responses relating to one cue, then another cluster related to a different cue, and so on until the correct response was identified. Such behavior was considered indicative of a sequential or serial consideration of each cue in turn.

Both Smith et al. (2013) and Davelaar (2015) aimed to gain a real-time insight into the memory-intersection process, via a novel manipulation requiring participants to make 
frequent responses during the problem solving. However, the response mechanism used to collect such responses is necessarily serial, in that only one word can be typed at a time. Even a parallel system can appear serial when funneled through a serial 'bottleneck' (see e.g., Pashler, 1994b, 1994a). In addition, the task design and response mechanism may have changed the way participants normally complete the task; participants 'candidate' responses might themselves have become 'cues', which would explain the sequential dependencies in the responses observed by both authors. Thus the novel design may have masked or even changed any potentially parallel process participants would generally use. A final concern is that parallel models can often produce the exact data patterns generated by a serial model (e.g., Townsend, 1972, 1990 ). Generally it is necessary to examine the full distribution of completion times for a given process to distinguish the two architectures. Both Smith et al. and Davelaar draw their conclusions from time-independent data, which may be insufficient to distinguish serial and parallel systems (Howard et al., 2017).

\section{Systems Factorial Technology}

For the above reasons, the question of serial vs parallel processing in memory intersection problems is still open. To resolve this question, we developed a novel task that allowed adaptation of Systems Factorial Technology (Townsend \& Nozawa, 1995) to the memory intersection paradigm. SFT is a theory driven methodology for the identification of perceptual and cognitive mechanisms. In particular, it provides a framework for distinguishing serial and parallel processes within cognitive systems (Townsend \& Wenger, 2004). There are several aspects of the methodology, but importantly for our dual-cue case, the salience of the cues (i.e., the strength in which each cue predicts, or is related to a target word) can be factorially manipulated such that the two cues are combined to create four conditions - High-High (HH), High-Low (HL), Low-High (LH), and Low-Low (LL), where High and Low refer to the salience level of each cue. In the current study, we defined cue salience by the associative strength between a cue and a target, as defined by 
normative data sets (De Deyne, Navarro, \& Storms, 2013). The Mean Interaction Contrast (MIC) can then be computed, such that $\mathrm{MIC}=\mathrm{MRT}_{H H}-\mathrm{MRT}_{H L}-\mathrm{MRT}_{L H}+\mathrm{MRT}_{L L}$, where $\mathrm{MRT}_{H H}$ is the mean RT of the HH condition. The sign of the MIC can be used to diagnose processing architecture. Further diagnostics can be obtained by substituting the survivor function of response times (where $\mathrm{S}(t)=1-\mathrm{F}(t)$; the complement of the cumulative distribution function of response time) in place of the mean response-times in the above formula. For a comprehensive treatment of SFT we direct readers to excellent tutorials by Houpt, Blaha, McIntire, Havig, and Townsend (2014) or Altieri et al. (2017) .

Recent Bayesian advances (see Houpt \& Fifić, 2017; Houpt, Heathcote, \& Eidels, 2017) allow evidence for and against serial/parallel processing to be directly computed within the SFT framework. As such, SFT is the ideal approach for the present study. We report a series of four experiments that implement a novel adaptation of SFT to the domain of semantic memory. All experiments had the same basic design, where two cue words were presented to probe an intersection process. In Experiments 1 and 2, participants were required to generate the intersection of these cues (by either typing or vocalizing the solution respectively), in Experiment $3 \mathrm{a}$ and $3 \mathrm{~b}$ participants were asked to judge whether a third 'candidate' word was the intersection.

In Experiment 1 we aimed to align our methodology as closely as possible to Smith et al. (2013). Thus we presented to participants two word cues that prompted them to type a third 'related' word as a response. In Experiment 2 we substituted typed responding for vocal responding to ensure 'response planning' was not contaminating the recorded response times. We present Experiments 1 and 2 with a common Method and Results. In Experiments $3 \mathrm{a}$ and $3 \mathrm{~b}$ we explored a novel yet related question of dual-cued recognition (as opposed to recall) to address a secondary objective ("are dual cues treated differently in recall vs recognition tasks"). To that end, in Experiments 3a and 3b we presented participants with a 'candidate' solution to the intersection problem, with a simple yes/no response requested. Over the course of these four experiments, manipulating both response 
mechanism (typing, vocal, and button press) and recall/recognition memory designs, we find the overwhelming majority of evidence favors a parallel processing strategy. This trend was strongest for recall tasks; recognition tasks also showed primarily parallel processing, but were more amenable to serial processing by some participants.

\section{Method - Experiments 1 and 2 (Recall)}

In the recall tasks, participants were presented with two cues and were required to generate a response that corresponded to the 'intersection' of those two cues. For example, given the two cues "billiards" and "chlorine" the correct response (to be typed or spoken aloud) was "pool". Across all of our experiments the intersections of cues were defined by taking words from De Deyne et al.'s (2013) free association norms that shared a single common response; as such, our target responses were defined by large-scale normative data and not pre-specified problems as in the RAT (Birren, 1964). Participants only responded once they had solved a problem, and this was done by either (a) typing the answer using a standard computer keyboard (Experiment 1), or (b) pressing the SPACEBAR key then vocalizing a response (Experiment 2). Response-time was captured at the first key press in both cases.

\section{Participants}

The study was approved by the Human Ethics Committee at the Unviersity of Newcastle. Participants ( $\mathrm{n}=29$ in Experiment 1, $\mathrm{n}=39$ in Experiment 2) were undergraduate psychology students at the University of Newcastle, Australia. Age ranged from 18-37 $(\mathrm{M}=22.3$ years, $\mathrm{SD}=4.82$ years $)$. Seven participants were male in each experiment, and all but one (from Experiment 1) were right handed.

\section{Stimuli}

Stimuli were triplets of English words, two of the words serving as cues and presented to the participants, and a third, target word that should have been generated by the 
participants if they were correct in solving the intersection challenge. Cue-Intersection sets were constructed from Free-Association norms reported by De Deyne et al. (2013), in which over 70,000 subjects were presented with a 'cue' word, and asked to freely generate up to three related words. Inappropriate words were filtered before stimulus selection proceeded. These included offensive words along with words that may have been particularly salient to the student population (e.g., the word 'depression' was excluded due to the participants being psychology students). To manipulate cue salience, we extracted forward-probabilities for each cue-response pairing (i.e. the proportion of subjects who responded 'cat' given the cue 'dog'). Low salience pairs were defined by a cue-target forward-probability between 0.25 - 0.35 in Experiment 1, and 0.15 - 0.3 in Experiment 2. High salience was defined as forward-probability between 0.45 - 0.55 in Experiment 1, and $0.4-0.8$ in Experiment 2.

To identify cue-cue-target triplets we extracted any two cues that shared a single common response, with each cue-response pairing also satisfying the associative strength constraints defined above. Only cue-pairs that shared a single intersection were chosen to avoid potential response conflicts and to ensure there was a clearly defined target. To reduce interference effects, we also limited our stimulus sets such that each 'target' word was unique (i.e., no two trials required the same response).

\section{Design}

Each trial consisted of a display containing two cue words, one above the other, as shown in Figure 2. In Experiment 1 a text-box was present under the cues; this was not required in Experiment 2. To allow estimation of the Mean and Survivor Interaction Contrasts, we incorporated a factorial manipulation of salience x cue location. Cue salience was defined by the forward probability between each cue and the target response. Cue-target association could be high or low (see Stimulus section) for each target, and factorially combining these manipulations resulted in a 2x2 set of High-High, High-Low, Low-High and Low-Low. An example High-Low trial would be the cues "dracula" and 
"stake" requiring the target response "vampire". "Dracula" had a higher forward probability $(\sim .55)$ to the target than "stake" ( $\sim 17)$, which should result in faster processing times for the stronger relationship (Houpt et al., 2014).

\section{Procedure}

Demographic information (age, sex, handedness) was collected, and participants provided informed consent before proceeding with the experiment. Participants were seated at a computer in the Newcastle Cognition Lab at the University of Newcastle in a dark room. Participants were instructed that throughout the experiment they would be presented with word pairs, and would need to generate a response related to both cue words. Participants were asked to respond as quickly and accurately as possible. The experiment was administered using Psychopy 1.82.1. In Experiment 1 twenty reading-typing trials were presented initially to allow post-hoc examination of the reading and typing components of response-time if necessary. In each of these trials, participants were presented with two unrelated words, and asked to type both words into the text-box. These data were not analyzed further. In Experiment 2 this block was replaced with a microphone and headset check with the Experimenter assisting with setting recording levels. 
(a)

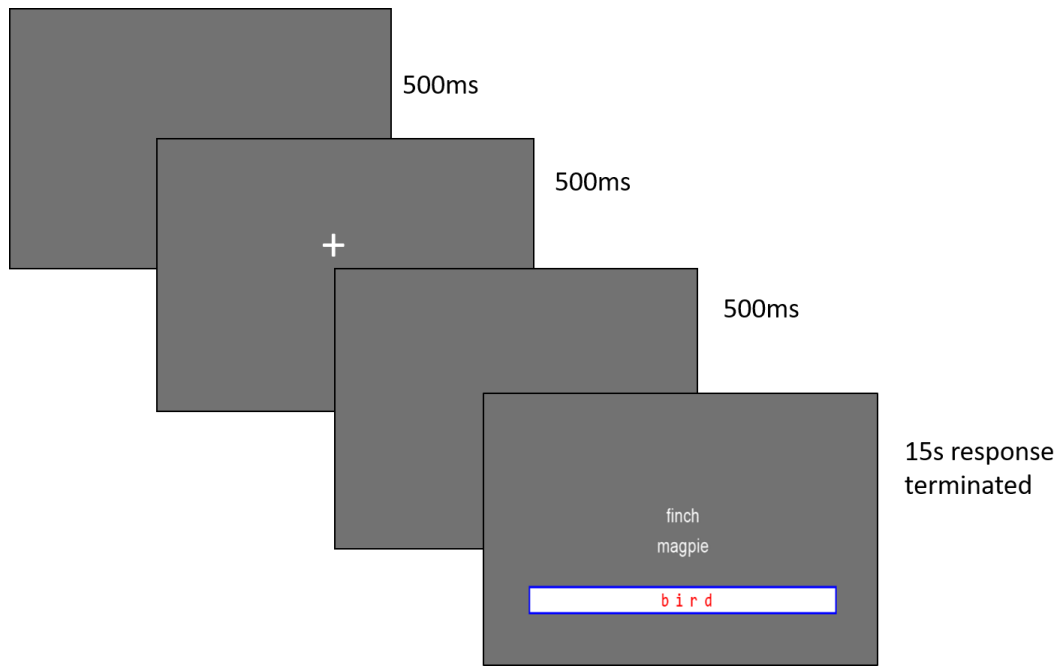

(b)

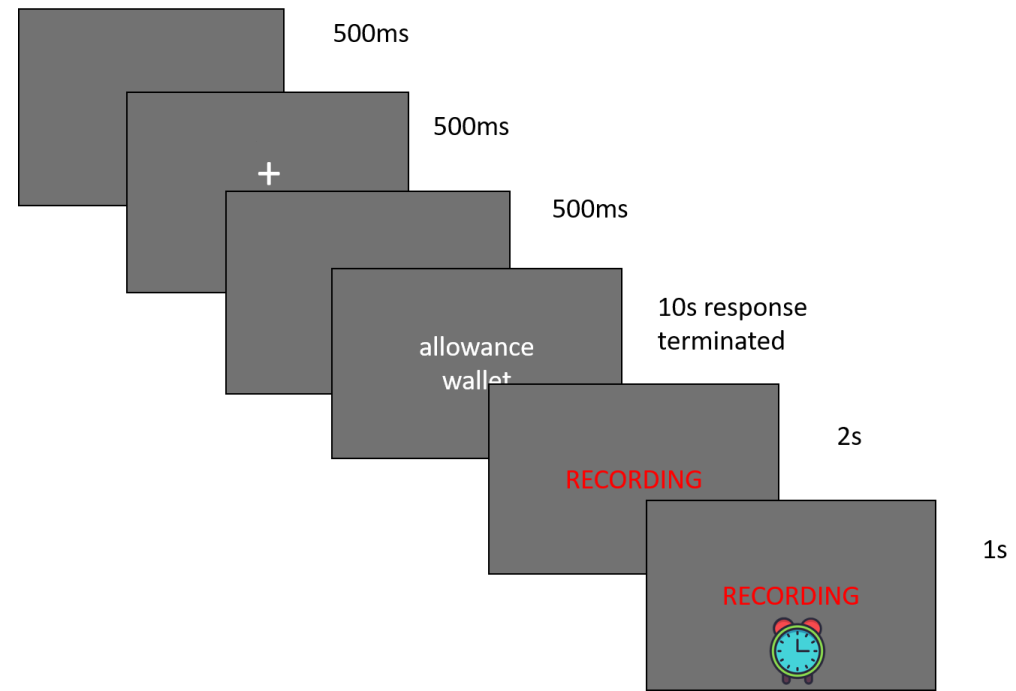

Figure 2. Illustration of a trial sequence in Experiment 1 (top) and 2 (bottom). Trials started with a $500 \mathrm{~ms}$ inter-stimulus-interval, followed by a $500 \mathrm{~ms}$ fixation at the center of the screen, then another 500ms blank before the simultaneous presentation of two cue words, one above and one below fixation. In Experiment 1, participants then had up to 15 seconds to view the cues, make a decision, and type their response (pressing the 'Enter' key to terminate the trial). In Experiment 2 the response time-window was 10 s and participants were instructed to press the 'SPACEBAR' key, then speak their response out loud within $3 \mathrm{~s}$.

Following the initial block, participants were given instructions regarding the main task. Fourteen practice trials that were identical to the main experiment were presented, except that these trials provided feedback. In Experiment 1 feedback was given in the form 
of "correct" or "incorrect", plus the correct response. In Experiment 2 real-time accuracy assessment was not possible, so instead the correct response was shown, along with the words "You said:", while a recording of the participants response played. For the main experimental trials, each trial started with a 500ms blank screen, 500ms fixation, and another 500ms blank screen. Following this, the two cue words were displayed on screen, and remained there until the participant either typed their response in Experiment 1 (indicating they were finished by pressing the ENTER key), or pressed the SPACEBAR key in Experiment 2. The trial timed out after 15s or 10s in Experiment 1 and 2 respectively. In Experiment 1 a total of eight blocks consisting of twelve trials were presented, for a total of 384 trials (96 per salience combination). In Experiment 2 this was increased to 100 trials for each of the salience conditions, for a total of 400 trials. Minor data pre-processing was performed after data collection, manually checking each and every typed response in Experiment 1 for obvious typing errors (e.g., if the word 'dog' was typed 'odg' this was corrected in the data). For Experiment 2 audio recordings of each trial were transcribed and checked for accuracy.

\section{Results - Recall}

\section{Analysis}

We applied recently developed Bayesian analyses to directly compare evidence for serial and parallel processing. We implemented both a non-parametric assessment of SIC (Houpt et al., 2017) and a parametric, model-selection based assessment of MIC (we modified the hierarchical approach developed by Houpt \& Fifić, 2017, see Appendix A for details). We combined the evidence from both of these analyses (which were always consistent) to classify each participants data as more closely resembling serial or parallel processing. Thus the classifications here are based on two independent Bayesian analyses assessing separate, but related, metrics of processing (MIC and SIC) using different analytic approaches. All results were also cross-referenced by visually inspecting the 
empirical SIC estimated directly from the data. Non-Parametric model probabilities were computed using the programming language R (R Core Team, 2014), and parametric model fits were performed using the computational language STAN (Carpenter et al., 2017). Descriptive Bayesian statistics were performed using JASP (JASP Team, 2018).

\section{Descriptive Statistics}

Twenty-nine subjects participated in Experiment 1. Of these, 6 subjects did not meet the minimum accuracy criteria of at least $50 \%$ in each salience condition and were excluded from further analysis. All 6 subjects failed due to below-criterion accuracy in the LL condition, with LL accuracy ranging from .38 to .49 $(\mathrm{M}=.45)$ for those subjects. An additional 4 subjects failed to meet the selective-influence requirement and were also excluded. Three of those subjects demonstrated a non-dominant relationship, with no clear salience effect at all, and the remaining subject showed a stochastic dominance relationship such that $S(t)_{\mathrm{LH}}<S(t)_{\mathrm{HH}}$, violating the requisite ordering of response-time distributions (Houpt et al., 2014). Of the thirty-nine participants that completed Experiment 2, twelve were excluded from further analysis due to failing the minimum accuracy criteria of at least $50 \%$ in each salience condition. All 12 subjects failed in the LL condition, with accuracy in that condition ranging from .31 to $.49(\mathrm{M}=.44)$ for those subjects.

The remaining subjects all showed the desired relationship between response-times distributions such that $S(t)_{\mathrm{HH}} \lesssim\left\{S(t)_{\mathrm{HL}}, S(t)_{\mathrm{LH}}\right\} \lesssim S(t)_{\mathrm{LL}}$, and a stochastic dominance relationship between $S(t)_{\mathrm{HH}}<S(t)_{\mathrm{LL}}$ with a Bayes Factor of at least 5. At the group level, the manipulation of salience clearly affected both mean response-time and accuracy, with RT increasing and accuracy decreasing from $\mathrm{HH} \rightarrow\{\mathrm{HL}, \mathrm{LH}\} \rightarrow$ LL, allowing valid consideration of the SFT results. All differences in both accuracy and RT were strong $B F_{10}>10$. 


\section{SFT Results}

The SFT results of all present Experiments are summarised in Figure 3. Of the 19 subjects analysed in Experiment 1, 17 showed evidence for parallel processing ( 5 exhibited evidence for self-terminating or co-activating processing, 12 for exhaustive processing). The remaining two participants exhibited evidence for serial processing. Of the 27 subjects analysed in Experiment 2, 18 showed evidence for parallel processing (1 exhibited evidence for self-terminating processing, 17 for exhaustive processing). One participant exhibited evidence for serial processing. In all cases the Non-Parametric test was consistent with the independently fit parametric test, which strengthens the conclusion that most subjects completed the task using a parallel process. LOOIC and posterior probabilities for each subject are included in Appendix B.

For 8 subjects in Experiment 2 there was no clearly preferred model, and these subjects could not be classified with confidence. For these subjects, visual inspection confirmed that none of the 5 exemplar SFT models were supported. It is possible that these data reflect some mixture of serial and parallel processing (see e.g., Little, Eidels, Houpt, Garrett, \& Griffiths, 2018; Tillman \& Evans, 2019), or a hybrid stopping rule however there is no available diagnostic for such phenomena at this time. 


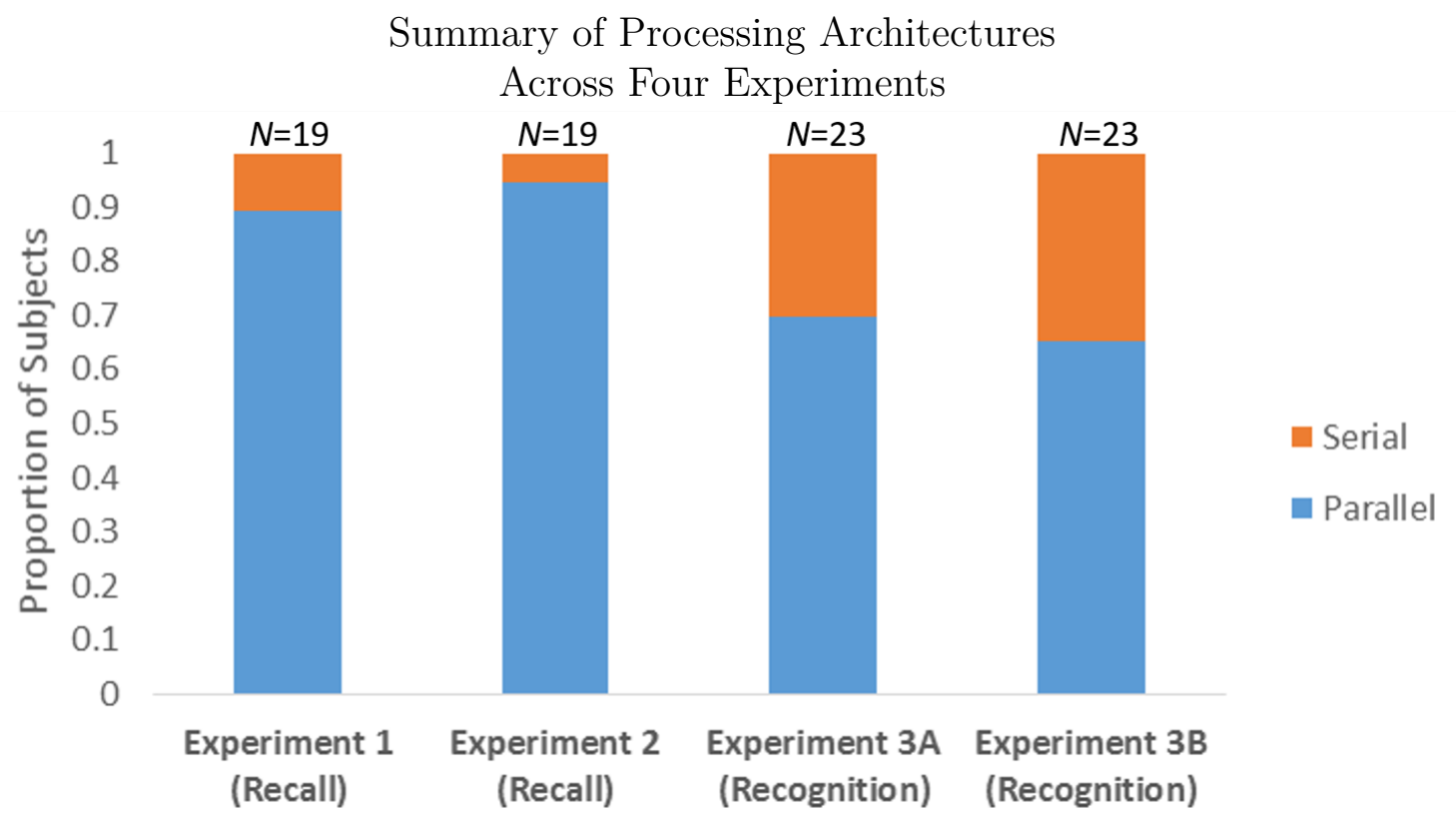

Figure 3. Summary of processing architecture results for each experiment. Almost all subjects whose data could be classified in Experiment 1 and 2 showed evidence for a parallel processing architecture. In Experiment 3 (A and B) the majority still showed parallel processing, but a subset of subjects showed evidence for serial processing. The number of total participants included in each graph are shown at the top of each bar. NB: 8 subjects in Experiment 2 could not be classified with sufficient evidence. As no inference can be drawn regarding these participants processing architecture they have been omitted from this summary figure.

\section{Interim Discussion}

The results of Experiments 1 and 2 were fairly conclusive. Of the 38 participants that could be classified across the two experiments, 35 exhibited converging evidence for parallel processing in a memory intersection task. Only 3 subjects showed evidence for a serial processing, and 8 could not be classified. These results contrast with the findings of Smith et al. (2013) and Davelaar (2015) as we expected. Our novel paradigm successfully introduced a cue-constrained memory task amenable to response-time investigations using SFT, whilst remaining as close as possible to the above studies, retaining the typed response mechanism in Experiment 1, and the generation of solution words in both experiments. There were several key differences between the tasks, with our design adapted to avoid interrupting the decision process before a final response had been generated, and 
focusing on speeded responses to generate response-times for analysis. We also presented only two cues, as opposed to the three cues presented by Smith et al. (2013) and Davelaar (2015) - this modification was necessary to utilize the diagnostic power of SFT.

It is not clear why some subjects in Experiment 2 did not exhibit any of the canonical SFT model signatures. It is possible that these subjects utilized some hybrid or mixture of processes, which can be difficult to diagnose (Little et al., 2018; Tillman \& Evans, 2019). Regardless, taken as a whole Experiments 1 and 2 provide replicated evidence that memory intersection problems can and usually are solved using a parallel process. This evidence comes from two different task designs (typed and spoken response), using two converging independent Bayesian analyses - one parametric and one non-parametric, using different participant pools and stimulus sets. Both of these tasks speak to memory recall; i.e. problems that require the generation of responses from memory.

Next, we test if processing within the same multiply-constrained framework is parallel or serial when applied to a recognition paradigm. Recognition is generally considered a much easier task (Anderson \& Bower, 1972); indeed it is often assumed that recognition is a subset of the recall process (e.g. Kintsch, 1970). Further, unlike recall tasks, which require a focused search and comparison process to identify a potential solution, it is not difficult to imagine a serial process arising from a recognition-based intersection problem. Such a problem would present participants with a 'candidate' answer, and ask whether that candidate was the intersection of two given cues. A simple comparison of the candidate to Cue A then Cue B sequentially could easily answer the problem. Therefore, it is theoretically possible that processing architecture differs across recall and recognition-based intersection problems.

To our knowledge, recognition style multiply-constrained problems have rarely been studied. However, they are likely as prevalent in everyday life as the more commonly studied recall/generative problems. For example, rather than generating a recipe based on the ingredients in our fridge, we may read through a recipe book to recognize one that can 
be made with the available ingredients. Alternatively, you may be solving a crossword with a friend, who asks you if the answer to three-down is "mayonnaise"? In these scenarios solving the problem still requires both cues to be considered, yet it is less clear that they necessarily be considered simultaneously. You may sequentially check off the ingredients list from the recipe book, and you would still determine the suitability of the dish.

To examine multiply-constrained search strategy across the recognition-recall distinction, in Experiment 3 we adapted the paradigm used in the first two experiments to a novel recognition framework. Participants were provided a candidate answer to an intersection problem, and were asked to determine whether it was a suitable solution. Responses were simple key presses corresponding to yes/no alternatives. This modification required the introduction of catch trials ('non-intersection'), i.e. trials in which the provided candidate was not the solution to the cues. The only difference between Experiment $3 \mathrm{a}$ and $3 \mathrm{~b}$ was the forward-probability values used in the salience manipulation. Further details of methodological changes are included below.

\section{Method - Experiments 3a and 3b (Recognition)}

\section{Participants}

Participants $(\mathrm{n}=50)$ were undergraduate psychology students at the University of Newcastle. Age ranged from 18-39 ( $\mathrm{M}=21.48$ years, $\mathrm{SD}=4.46$ years). Nine participants were male, and all but one were left handed.

\section{Stimuli}

The preparation of stimulus-triplet was the same as Experiments 1 and 2. For Experiment 3 we generated two different stimulus sets, one with a wider separation of association strengths but potentially more difficult (due to the very low association strength of the low-salience range). In Experiment 3a, low-salience pairs were defined by a cue-target association strength of between 0.03 and 0.08 . High-salience was defined as 0.40 
to 0.80. In Experiment 3b, low-salience association strength ranged from 0.05 to 0.10 , and high salience ranged from 0.35 to 0.60. As the design for Experiment 3 incorporated both 'yes' and 'no' responses, non-intersection stimuli were also created. These trials could have either one or neither cue related to the target word. To generate trials where only one cue was related to the target we generated additional 'intersection' trials, then shuffled one cue list, manually checking that this did not result in an additional intersection. For trials where both cues were unrelated to the target triplets were pulled at random from the database and again manually checked to ensure there were no randomly-selected relationships.

\section{Design}

The design of Experiment 3 differed slightly from the first two experiments. Two cues were presented in identical fashion as the earlier experiments, with the caveat that they were not always related to the target. Whereas previously participants were required to generate a target related to cue cues, in Experiment 3 they were presented a third, target word and asked whether the provided word was semantically related to both cues. This word was presented on a separate screen after the cue presentation, and was displayed in yellow font to distinguish it from the cues. This change meant cues were no longer displayed for the entire duration of the trial as they had been in earlier Experiments.

\section{Procedure}

Demographic information (age, sex, handedness) was collected and participants attended the Newcastle Cognition Lab at the University of Newcastle to complete the experiment. Participants provided informed consent before proceeding, and were then randomly pre-assigned to Experiment 3a or 3b (which were identical apart from stimuli), and the location of response keys was also counterbalanced between participants. Two subjects from Experiment 3a did not attend their session. Participants were told they would be presented with word triplets: two cue words presented vertically on the screen, 
followed by a target word on the following screen. Participants were instructed to press the key marked with a blue sticker if both cue words were related to the target word, and the key marked with the yellow sticker if either or both cues were unrelated to the target word. Participants were instructed to respond as quickly and accurately as possible.

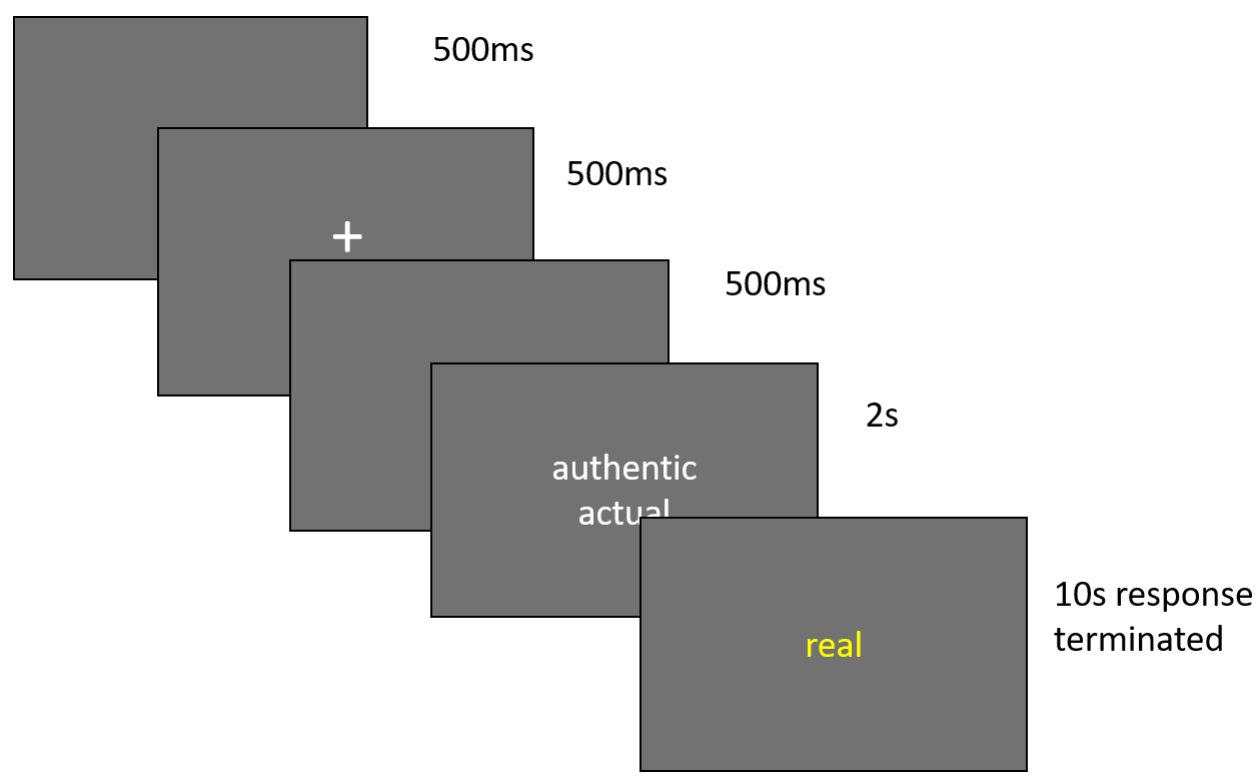

Figure 4. Illustration of a trial sequence in Experiment 3. Between trials there was a $500 \mathrm{~ms}$ inter-stimulus-interval, followed by a $500 \mathrm{~ms}$ fixation at the center of the screen, then another 500ms blank before the cue words were presented. Participants then had 2 seconds to view the cues, after which the candidate response was displayed for up to $10 \mathrm{~s}$.

Thirty practice trials were presented to familiarize participants with the task. The association strengths for these trials approximated the higher salience values used in the experiment, but the word triplets were different. The experiment was administered using Psychopy 1.82.1. On each practice trial, a fixation cross was presented for $500 \mathrm{~ms}$, followed by two cues presented on the screen for $2 \mathrm{~s}$ (to allow sufficient time for even the longest cues to be read), and finally the target word appeared in yellow font until a response was made. During practice trials, participants received correct/incorrect feedback after each trial. An example trial sequence is provided in Figure 4.

After completing the practice block participants began the experimental trials. These were identical in structure to the practice trials, except that no feedback was given. A time 
limit of 10 seconds was imposed, after which the words "TOO SLOW!" were shown followed by the next trial. A total of 10 blocks of 58 trials were presented, with enforced breaks of at least 30 seconds between blocks to reduce fatigue. Of the 580 trials, $70 \%$ (400) of the cue pairs were both related to the target item with an equal number of trials (100) in each salience combination (HH, HL, LH, and LL). The other 180 trials were evenly divided (60 trials each) among the remaining three conditions - single related cue on top, single cue at the bottom, and trials where neither cue was related to the target. Salience levels and relatedness were randomly presented throughout the experiment.

\section{Results - Experiment 3}

\section{Descriptive Statistics}

Fifty subjects participated in Experiment 3 - 24 in Experiment 3a and 26 in Experiment 3b. Of these, 4 subjects in total were excluded from further analysis as they did not meet the minimum accuracy criteria of at least $50 \%$ in each salience condition, one subject from Experiment 3a and three from Experiment 3b. As 'non-intersection' trials are not pertinent to the question of processing architecture they are not considered further, but were included in the accuracy exclusions checks. The remaining subjects all showed a relationship between response-times such that $S(t)_{\mathrm{HH}} \lesssim\left\{S(t)_{\mathrm{HL}}, S(t)_{\mathrm{LH}}\right\} \lesssim S(t)_{\mathrm{LL}}$, and a stochastic dominance relationship between $S(t)_{\mathrm{HH}}<S(t)_{\mathrm{LL}}$ with a Bayes Factor of at least 5. At the group level, the manipulation of salience clearly affected both mean response-time and accuracy, with RT increasing and accuracy decreasing from $\mathrm{HH} \rightarrow$ $\{$ HL,LH $\} \rightarrow$ LL. All differences in both accuracy and RT were strong $B F_{10}>10$.

\section{SFT Results}

Of the 23 subjects considered for analysis in Experiment 3a, 16 showed converging evidence for parallel processing ( 1 exhibited evidence for self-termination, 15 for exhaustive processing). The remaining seven participants exhibited evidence for serial processing. In 
3b the numbers were similar, with 15 participants showing evidence for parallel processing (1 self-terminating, 14 exhaustive), and the remaining 8 showing evidence for serial processing. These results are summarized alongside the other Experiments in Figure 3. In all cases the non-parametric test was consistent with the parametric test. LOOIC and posterior probabilities for each subject are included in Appendix B

\section{General Discussion}

The results of Experiment 3 are generally consistent with Experiments 1 and 2, in that the majority of subjects showed evidence for parallel processing. However, a subset $(\sim 33 \%)$ in Experiments $3 \mathrm{a}$ and $3 \mathrm{~b}$ showed evidence for a serial processing architecture. This trend was remarkably consistent between Experiments 3a and 3b, and highlights a distinct strategy difference between recall and recognition based intersection tasks. We speculated earlier that a recognition task may be more amenable to serial processing than an equivalent recall task, and it is interesting that such distinction was observed in our data. To our knowledge this processing distinction represents a novel contribution to the field of constrained memory, and warrants further experimental and theoretical consideration.

Experiments 1 and 2 provide strong evidence for parallel processing, showing almost all participants utilized a parallel architecture when considering memory problems requiring the integration of two cues to generate a response from recall memory. This result is at odds with recent work (Smith et al., 2013; Davelaar, 2015), but consistent with earlier theoretical perspectives (e.g., Rubin \& Wallace, 1989). Indeed, parallel operation of memory processes in general is commonly posited. For example, Humphreys, Tehan, O'Shea, and Bolland (2000) argued that interference from other list items in a recall task is necessarily the result of parallel access pathways to the target word(s). Logan and Schulkind (2000) presented evidence that participants can retrieve information about two different cues simultaneously in a semantic memory task, and Logan and Delheimer (2001) 
extended this finding to episodic and lexical memory retrieval as well. Both the Spreading Activation (Collins \& Loftus, 1975) and Compound Cue (McKoon \& Ratcliff, 1989, 1992) accounts of priming effects rely on parallel activation of items in long term memory. In terms of dual-cue memory tasks, both Wenger (1999) and Rickard and Bajic (2004) found that cues could be used simultaneously, although these tasks were not constrained such that both cues were required to be processed.

Parallel processing has long been the default assumption in other types of multiply constrained problem solving. Kunda and Thagard (1996) argued that people form impressions about others by simultaneously integrating stereotypes and individuating information to perform 'constraint satisfaction'. Many authors have assumed that the Remote Associates Task (Mednick, 1968), one of the leading examples of multiply constrained retrieval in a lab setting (and the task adapted by Smith et al., 2013), is solved by a parallel spreading activation process in which the target is activated several times (e.g. Bolte et al., 2003; Topolinski \& Strack, 2008). These theoretical perspectives are supported by our data, replicated across several task designs and converging analysis. So why are these results at odds with Smith et al. (2013) and Davelaar (2015)?

As noted in the introduction, the novel methodological changes introduced by Smith et al. (2013) may have inadvertently influenced the observed processing strategy. First, it is difficult to conceptualize how a sequence of candidate words generated by a participant would clearly indicate a parallel process. A sequence of words that were truly independent from each other, only drawn from the cues, could be explained equally well by a parallel model, or a serial model switching between cues after each candidate. A sequence of sequentially dependent candidates (such as those observed by Smith et al., 2013) could simply be the result of a parallel process being 'bottlenecked' by a serial response process (Pashler, 1994a). More alarmingly, the response process itself could have influenced the decision process. Given the serial nature of writing down candidate words participants may have been encouraged to consider cues serially. They may also have used the candidate 
words they generated as cues to new candidates, which would explain the sequential dependence, or 'clustering' of responses. There is also the distinct possibility that the frequent typing of candidate answers constitutes an 'interruption' of the decision process, which entails a host of implications that question the generalizability of the findings (see e.g., Salvucci, 2010).

The model proposed by Smith et al. (2013) to explain the behavior of participants in their study is similar in several key aspects to a model proposed by Mueller and Thanasuan (2013) to explain crossword solving. Crosswords are possibly the canonical example for problem-solving processes that require the integration of multiple constraints, so it is intriguing that a serial model should be proposed in that domain as well. Mueller and Thanasuan (2013) proposed a model in which crossword problems are solved by sampling candidate responses from one cue (typically the semantic, or content cue) and then comparing this candidate to the additional constraints - the number of letters, and any letters pre-filled from other responses. They found that their (serial) model provided a better account of both novice and expert crossword solver's data than a model that simultaneously considered both constraints. While this finding seems inconsistent with our present results, there are several observations that could resolve the conflict. First, in a crossword puzzle, it is almost always the case that one cue corresponds to the meaning of the target, and one is the number of letters. In our task the dimensions are generally all semantic or phonological properties of the targets. It is possible that the number of letters is psychologically separable from meaning, favouring a serial search (Little, Nosofsky, Donkin, \& Denton, 2013), whereas the meaning/phonological properties of targets in our task are more naturally suited to a parallel strategy (Fific, Little, \& Nosofsky, 2010; Little et al., 2013). It is also plausible that crossword puzzles are simply more difficult, which has also been shown to occasionally change processing from parallel to serial (Luria \& Meiran, 2005). We believe our results can co-exist with Mueller and Thanasuan's without issue.

Our results target a broad class of model frameworks, rather than a specific 
formulation. In this way, like Wenger (1999), our contribution should shape more fine-grained investigation of specific process models in the future. Our data strongly suggest intersection problems requiring a generative solution typically involve a parallel consideration of the cues or constraints of the problem - future models should therefore incorporate this kind of architecture. Where a candidate answer is provided the process can be, but is not always parallel, and future theoretical developments may propose mechanisms to account for these differences in processing. Our results reinforce the arguments raised by Townsend (1972), Townsend (1990) and Townsend and Wenger (2004) among many others, that when considering the dynamic attributes of a processing system (e.g. the serial vs parallel nature of the process) model mimicry is a major concern and specialized techniques are needed to account for it.

\section{Conclusion}

Theories of memory almost exclusively focus on the retrieval of complete memory traces - the remnants of coherent experiences. However, people are not restricted to endlessly recapitulating the past. They combine cues in novel ways to generate new solutions. The intersection process is a building block of this creative process and understanding its properties is fundamental to capturing the generativity of human cognition. We provide strong evidence across a number of experiments that the overwhelming majority of participants generate responses to intersection problems by considering the constraints, or cues, in parallel. In recognition tasks we found a similar trend, yet a subset of participants in both Experiments 3a and 3b utilised a serial strategy, hinting at a theoretically meaningful distinction between the approaches to the two tasks. These results provide valuable insight into memory processes that can be incorporated into future theoretical developments. 


\section{References}

Altieri, N., Fifić, M., Little, D. R., \& Yang, C.-T. (2017). Historical foundations and a tutorial introduction to systems factorial technology. In Systems factorial technology (pp. 3-25). Elsevier.

Anderson, J. R., \& Bower, G. H. (1972). Recognition and retrieval processes in free recall. Psychological review, $79(2), 97$.

Birren, J. E. (1964). The psychology of aging. Prentice Hall.

Bolte, A., Goschke, T., \& Kuhl, J. (2003). Emotion and intuition: Effects of positive and negative mood on implicit judgments of semantic coherence. Psychological Science, $14(5), 416-421$.

Britannica, E. (1965). Encyclopaedia britannica. Inc., chicago.

Carpenter, B., Gelman, A., Hoffman, M. D., Lee, D., Goodrich, B., Betancourt, M., ... Riddell, A. (2017). Stan: A probabilistic programming language. Journal of statistical software, $76(1)$.

Collins, A. M., \& Loftus, E. F. (1975). A spreading-activation theory of semantic processing. Psychological review, 82(6), 407.

Craik, F. I. (2002). Levels of processing: Past, present... and future? Memory, 10(5-6), $305-318$.

Davelaar, E. J. (2015). Semantic search in the remote associates test. Topics in cognitive science, $7(3), 494-512$.

De Deyne, S., Navarro, D. J., \& Storms, G. (2013). Better explanations of lexical and semantic cognition using networks derived from continued rather than single-word associations. Behavior research methods, 45(2), 480-498.

Fific, M., Little, D. R., \& Nosofsky, R. M. (2010). Logical-rule models of classification response times: A synthesis of mental-architecture, random-walk, and decision-bound approaches. Psychological Review, $117(2), 309$.

Frey, P. W., \& Adesman, P. (1976). Recall memory for visually presented chess positions. 
Memory \& Cognition, 4(5), 541-547.

Heathcote, A., Brown, S., Wagenmakers, E., \& Eidels, A. (2010). Distribution-free tests of stochastic dominance for small samples. Journal of Mathematical Psychology, 54(5), $454-463$.

Houpt, J. W., Blaha, L. M., McIntire, J. P., Havig, P. R., \& Townsend, J. T. (2014). Systems factorial technology with r. Behavior research methods, 46(2), 307-330.

Houpt, J. W., \& Fifić, M. (2017). A hierarchical bayesian approach to distinguishing serial and parallel processing. Journal of Mathematical Psychology, 79, 13-22.

Houpt, J. W., Heathcote, A., \& Eidels, A. (2017). Bayesian analyses of cognitive architecture. Psychological methods, 22(2), 288.

Houpt, J. W., \& Townsend, J. T. (2010). The statistical properties of the survivor interaction contrast. Journal of Mathematical Psychology, 54 (5), 446-453.

Howard, Z. L., Eidels, A., Silbert, N. H., \& Little, D. R. (2017). Can confusion-data inform sft-like inference? a comparison of sft and accuracy-based measures in comparable experiments. In Systems factorial technology (pp. 291-317). Elsevier.

Humphreys, M. S., Tehan, G., O’Shea, A., \& Bolland, S. W. (2000). Target similarity effects: Support for the parallel distributed processing assumptions. Memory \& Cognition, 28(5), 798-811.

JASP Team. (2018). JASP (Version 0.9)[Computer software]. Retrieved from https://jasp-stats.org/

Kintsch, W. (1970). Models for free recall and recognition. Models of human memory, $331-373$.

Kunda, Z., \& Thagard, P. (1996). Forming impressions from stereotypes, traits, and behaviors: A parallel-constraint-satisfaction theory. Psychological review, 103(2), 284.

Little, D. R., Altieri, N., Fific, M., \& Yang, C.-T. (2017). Systems factorial technology: A theory driven methodology for the identification of perceptual and cognitive 
mechanisms. Academic Press.

Little, D. R., Eidels, A., Houpt, J. W., Garrett, P. M., \& Griffiths, D. (2018). Systems factorial technology analysis of mixture models. Journal of Mathematical Psychology.

Little, D. R., Nosofsky, R. M., Donkin, C., \& Denton, S. E. (2013). Logical rules and the classification of integral-dimension stimuli. Journal of Experimental Psychology: Learning, Memory, and Cognition, 39(3), 801.

Logan, G. D., \& Delheimer, J. A. (2001). Parallel memory retrieval in dual-task situations: Ii. episodic memory. Journal of Experimental Psychology: Learning, Memory, and Cognition, 27(3), 668.

Logan, G. D., \& Schulkind, M. D. (2000). Parallel memory retrieval in dual-task situations: I. semantic memory. Journal of Experimental Psychology: Human Perception and Performance, 26(3), 1072.

Luria, R., \& Meiran, N. (2005). Increased control demand results in serial processing: Evidence from dual-task performance. Psychological Science, 16(10), 833-840.

Massaro, D. W., Weldon, M. S., \& Kitzis, S. N. (1991). Integration of orthographic and semantic information in memory retrieval. Journal of Experimental Psychology: Learning, Memory, and Cognition, 17(2), 277.

McKoon, G., \& Ratcliff, R. (1989). Assessing the occurrence of elaborative inference with recognition: Compatibility checking vs compound cue theory. Journal of Memory and Language, 28(5), 547.

McKoon, G., \& Ratcliff, R. (1992). Spreading activation versus compound cue accounts of priming: Mediated priming revisited. Journal of Experimental Psychology: Learning, Memory, and Cognition, 18(6), 1155.

Mednick, S. A. (1968). The remote associates test. The Journal of Creative Behavior, $2(3), 213-214$.

Mueller, S. T., \& Thanasuan, K. (2013). Model of constrained knowledge access in crossword puzzle players. In Proceedings of the 2013 international conference on 
cognitive modeling (iccm12).

Neisser, U. (2014). A review of ecological findings. In M. A. Conway, S. E. Gathercole, \& C. Cornoldi (Eds.), Theories of memory ii (p. 171-187). Oxford: Psychology Press.

Pashler, H. (1994a). Dual-task interference in simple tasks: data and theory. Psychological bulletin, $116(2), 220$.

Pashler, H. (1994b). Graded capacity-sharing in dual-task interference? Journal of Experimental Psychology: Human Perception and Performance, 20(2), 330.

R Core Team. (2014). R: A language and environment for statistical computing [Computer software manual]. Vienna, Austria. Retrieved from http://www.R-project.org/

Rickard, T. C., \& Bajic, D. (2004). Memory retrieval given two independent cues: Cue selection or parallel access? Cognitive Psychology, 48(3), 243-294.

Robbennolt, J., MacCoun, R., \& Darley, J. (2010). Multiple constraint satisfaction in judging. The psychology of judicial decision making, 27-40.

Rubin, D. C. (1995). Memory in oral traditions: The cognitive psychology of epic, ballads, and counting-out rhymes. Oxford University Press on Demand.

Rubin, D. C., \& Wallace, W. T. (1989). Rhyme and reason: Analyses of dual retrieval cues. Journal of Experimental Psychology: Learning, Memory, and Cognition, 15(4), 698.

Salvucci, D. D. (2010). On reconstruction of task context after interruption. In Proceedings of the sigchi conference on human factors in computing systems (pp. 89-92).

Smith, K. A., Huber, D. E., \& Vul, E. (2013). Multiply-constrained semantic search in the remote associates test. Cognition, 128(1), 64-75.

Taraban, R., \& McClelland, J. L. (1990). Parsing and comprehension: A multiple-constraint view. Comprehension processes in reading, 231-263.

Thomson, D. M., \& Tulving, E. (1970). Associative encoding and retrieval: Weak and strong cues. Journal of experimental psychology, 86(2), 255.

Tillman, G., \& Evans, N. J. (2019). Hierarchical bayesian mixture models of processing architectures and stopping rules. Journal of Mathematical Psychology. 
Topolinski, S., \& Strack, F. (2008). Where thereâẮ́sa willâĂŤthereâĂŹs no intuition. the unintentional basis of semantic coherence judgments. Journal of Memory and Language, 58(4), 1032-1048.

Townsend, J. T. (1972). Some results concerning the identifiability of parallel and serial processes. British Journal of Mathematical and Statistical Psychology, 25(2), 168-199.

Townsend, J. T. (1990). Serial vs. parallel processing: Sometimes they look like tweedledum and tweedledee but they can (and should) be distinguished. Psychological Science, 1(1), 46-54.

Townsend, J. T., \& Nozawa, G. (1995). Spatio-temporal properties of elementary perception: An investigation of parallel, serial, and coactive theories. Journal of Mathematical Psychology, 39(4), 321-359.

Townsend, J. T., \& Nozawa, G. (1997). Serial exhaustive models can violate the race model inequality: Implications for architecture and capacity. Psychological Review, $104(3), 595$.

Townsend, J. T., \& Wenger, M. J. (2004). The serial-parallel dilemma: A case study in a linkage of theory and method. Psychonomic Bulletin \& Review, 11(3), 391-418.

Treisman, A. M., \& Gelade, G. (1980). A feature-integration theory of attention. Cognitive psychology, 12(1), 97-136.

Tulving, E., \& Osler, S. (1968). Effectiveness of retrieval cues in memory for words. Journal of experimental psychology, r77(4), 593.

Tulving, E., \& Pearlstone, Z. (1966). Availability versus accessibility of information in memory for words. Journal of Verbal Learning and Verbal Behavior, 5(4), 381-391.

Tulving, E., \& Thomson, D. M. (1973). Encoding specificity and retrieval processes in episodic memory. Psychological review, 80(5), 352.

Vehtari, A., Gelman, A., \& Gabry, J. (2017). Practical bayesian model evaluation using leave-one-out cross-validation and waic. Statistics and Computing, 27(5), 1413-1432. 
Watkins, M. J., \& Tulving, E. (1975). Episodic memory: When recognition fails. Journal of experimental psychology: General, $104(1), 5$.

Wenger, M. J. (1999). On the whats and hows of retrieval in the acquisition of a simple skill. Journal of Experimental Psychology: Learning, Memory, and Cognition, 25(5), 1137. 
Appendix A

SFT Analysis

Traditionally, SFT studies have been high-accuracy (>90\%), simple perceptual studies with relatively large trial numbers. Our study, by necessity, had both lower accuracy and lower trial numbers. To account for this, we utilized Bayesian analysis techniques appropriate for smaller samples. To assess stochastic dominance (i.e., check the effect of the salience manipulation needed to propel SFT analysis), the standard Kolmogorov-Smirnov tests (Houpt \& Townsend, 2010) were not appropriate, given their low power at small sample sizes (see e.g., Heathcote, Brown, Wagenmakers, \& Eidels, 2010), so we instead implemented equivalent checks using the distribution free 'encompassing-prior' test proposed by Heathcote et al. which is appropriate for small samples $(\sim 50)$. We used a minimum criteria of $50 \%$ accuracy in all salience conditions to ensure we had sufficient trials to assess the manipulations - noting that a correct response in our experiments can almost certainly not result from guessing.

To obtain a complete account of our data we report a Bayesian assessment of both SIC and MIC separately to provide converging evidence for processing architectures, accounting for the uncertainty introduced by our sample size in different ways. We first implemented the non-parametric Dirichlet Process method proposed by Houpt et al. (2017). This technique relies on a non-parametric sampling process based on the observed distribution of response times, conceptually akin to a Bayesian bootstrapping procedure. On each posterior sample the 'sampled' distributions are classified as one of the five canonical SFT models (parallel exhaustive, parallel self-terminating, serial exhaustive, serial self-terminating, co-active) based on the combined evidence of the SIC and MIC of that sample, or classified as not matching any model. The evidence for each model is then weighed to give a posterior-probability for each possible SIC shape. In contrast to Houpt et al. we used a flat prior distribution across SIC shapes. This set the prior probability of any SIC shape equally likely and allowed an unbiased assessment of parallel vs serial models. 
To concurrently assess MIC, we adapted a modeling approach proposed by Houpt and Fifić (2017). ${ }^{1}$ Our model was fit to individual-subject level data, simultaneously fitting three 'MIC states'; positive, negative and zero. We used the same MIC-state model as Houpt and Fifić, with log-normal distributions (defined by $\mu$ and $\sigma$ parameters) to approximate the response time distributions (primarily to improve efficiency of the fitting process compared with their gamma distributions). We allowed the three MIC state models to fit independently, apart from shared $\sigma$ parameters for each response-time distribution. We then applied Leave One Out (LOO) cross-validation as recommended by Vehtari, Gelman, and Gabry (2017) to select the model that best explained the data for each subject.

The results for all experiments combine the posterior probability values for each SFT model with Leave One Out Information Criteria (LOOIC) values corresponding to three MIC states (positive, zero, negative). Because the focus of our investigation was architecture (serial vs parallel), not stopping rule (which should have been exhaustive for adequate performance), we combined SIC evidence for serial self-terminating and serial-exhaustive models, and likewise combined evidence for co-active and parallel self-terminating processes (which both assume parallel processing of cues). Thus for each analysis we have evidence for a parallel-self terminating (positive), serial (zero), or parallel exhaustive (negative) process. Subjects are classified as 'parallel' if a parallel model is selected by the LOO analysis, and the SIC results favor a parallel/coactive architecture with a posterior probability of at least 0.5 (implying the model is more likely than all alternatives combined), and vice-versa for serial processing. Where the evidence is weak for all models, the subject is classified as having 'inconclusive evidence' (this only occurred in Experiment 2).

\footnotetext{
${ }^{1}$ Note: We initially applied the full hierarchichal model proposed by these authors, however the results of the analysis suggested the group level was exerting too much influence and masking the individual variability in our data. These issues could not be resolved even in consultation with the original authors.
} 
Appendix B

Modelling Output

Non-Parametric

\begin{tabular}{l|lll|lll}
\hline Subject & Positive & Serial & Negative & \multicolumn{1}{c}{$>\mathbf{0}$} & \multicolumn{1}{c}{$=\mathbf{0}$} & \multicolumn{1}{c}{$<0$} \\
\hline 1 & 0.13 & $\mathbf{0 . 6 3}$ & 0.24 & 4269.812 & $\mathbf{4 2 6 8 . 2 8 5}$ & 4268.829 \\
2 & $\mathbf{0 . 8 1}$ & 0.19 & 0.00 & $\mathbf{4 4 0 8 . 2 7 3}$ & 4413.724 & 4419.058 \\
3 & $\mathbf{0 . 5 9}$ & 0.31 & 0.10 & $\mathbf{4 9 2 1 . 0 5 3}$ & 4921.12 & 4921.646 \\
4 & 0.00 & 0.22 & $\mathbf{0 . 7 7}$ & 4529.396 & 4525.949 & $\mathbf{4 5 2 3 . 8 2}$ \\
8 & 0.06 & 0.21 & $\mathbf{0 . 7 3}$ & 4048.167 & 4046.763 & $\mathbf{4 0 4 6 . 0 3 2}$ \\
12 & 0.05 & 0.32 & $\mathbf{0 . 6 2}$ & 5081.498 & 5079.638 & $\mathbf{5 0 7 8 . 8 1 9}$ \\
13 & 0.00 & 0.20 & $\mathbf{0 . 8 0}$ & 4537.727 & 4534.182 & $\mathbf{4 5 3 1 . 4 9 4}$ \\
15 & $\mathbf{0 . 8 1}$ & 0.18 & 0.01 & $\mathbf{4 1 8 3 . 5 6 6}$ & 4185.69 & 4188.87 \\
16 & 0.07 & 0.33 & $\mathbf{0 . 6 0}$ & 4836.057 & 4834.358 & $\mathbf{4 8 3 3 . 7 0 2}$ \\
17 & $\mathbf{0 . 5 3}$ & 0.36 & 0.10 & $\mathbf{4 0 6 9 . 3 6 4}$ & 4069.86 & 4071.247 \\
19 & 0.03 & 0.39 & $\mathbf{0 . 5 9}$ & 3427.537 & 3425.093 & $\mathbf{3 4 2 4 . 2 7 2}$ \\
20 & 0.05 & 0.19 & $\mathbf{0 . 7 7}$ & 4883.597 & 4882.179 & $\mathbf{4 8 8 1 . 1 4 1}$ \\
26 & 0.16 & 0.28 & $\mathbf{0 . 5 6}$ & 3886.446 & 3885.492 & $\mathbf{3 8 8 4 . 9 4 7}$ \\
27 & 0.07 & 0.29 & $\mathbf{0 . 6 3}$ & 4447.738 & 4446.376 & $\mathbf{4 4 4 5 . 7 9 4}$ \\
30 & 0.05 & 0.24 & $\mathbf{0 . 7 0}$ & 4771.064 & 4769.5 & $\mathbf{4 7 6 8 . 5 4 3}$ \\
31 & $\mathbf{0 . 6 2}$ & 0.36 & 0.02 & $\mathbf{3 7 3 2 . 1 7 4}$ & 3733.356 & 3736.133 \\
32 & 0.01 & 0.16 & $\mathbf{0 . 8 3}$ & 4304.662 & 4301.703 & $\mathbf{4 2 9 9 . 5}$ \\
33 & 0.00 & 0.12 & $\mathbf{0 . 8 7}$ & 4621.092 & 4617.593 & $\mathbf{4 6 1 5 . 1 1 4}$ \\
40 & 0.07 & $\mathbf{0 . 5 3}$ & 0.41 & 4659.114 & $\mathbf{4 6 5 6 . 8 0 1}$ & 4657.012 \\
\hline Table B1 & & & & & &
\end{tabular}

Table B1

Non-Parametric SIC posterior probabilities and parametric MIC model LOOIC scores for each subject in Experiment 1. SIC results have been combined to match the architecture output of the MIC states. Winning models for each method have been bolded for clarity. In all cases the two models provide converging evidence for the same processing architecture within a participant. 
Non-Parametric

\begin{tabular}{l|lll|lll}
\hline Subject & Positive & Serial & Negative & \multicolumn{1}{c}{$>\mathbf{0}$} & \multicolumn{1}{c}{$\mathbf{0}$} & \multicolumn{1}{c}{$\mathbf{0}$} \\
\hline 1 & 0.02 & 0.12 & $\mathbf{0 . 8 6}$ & 4574.075 & 4572.542 & $\mathbf{4 5 7 1 . 2 6 2}$ \\
2 & 0.45 & 0.38 & 0.17 & 5049.044 & 5048.815 & 5049.301 \\
3 & 0.07 & 0.32 & $\mathbf{0 . 6 1}$ & 4659.256 & 4657.835 & $\mathbf{4 6 5 7 . 1 8 8}$ \\
5 & 0.00 & 0.03 & $\mathbf{0 . 9 7}$ & 5355.543 & 5352.898 & $\mathbf{5 3 5 0 . 3 4 1}$ \\
7 & 0.01 & 0.21 & $\mathbf{0 . 7 8}$ & 4982.898 & 4980.114 & $\mathbf{4 9 7 8 . 5 7 9}$ \\
8 & 0.05 & 0.20 & $\mathbf{0 . 7 4}$ & 5741.21 & 5740.054 & $\mathbf{5 7 3 9 . 2 9 6}$ \\
10 & 0.48 & 0.39 & 0.13 & 4617.641 & 4617.699 & 4618.571 \\
11 & 0.07 & 0.30 & $\mathbf{0 . 6 4}$ & 4771.133 & 4769.459 & $\mathbf{4 7 6 8 . 9 6 1}$ \\
12 & 0.02 & 0.16 & $\mathbf{0 . 8 2}$ & 5150.228 & 5148.264 & $\mathbf{5 1 4 6 . 9 6 4}$ \\
13 & 0.05 & 0.27 & $\mathbf{0 . 6 8}$ & 5705.498 & 5703.628 & $\mathbf{5 7 0 3 . 0 8 4}$ \\
14 & 0.01 & 0.11 & $\mathbf{0 . 8 8}$ & 4783.803 & 4782.282 & $\mathbf{4 7 8 0 . 9 5}$ \\
15 & 0.05 & 0.25 & $\mathbf{0 . 7 0}$ & 5555.05 & 5553.318 & $\mathbf{5 5 5 2 . 4 0 3}$ \\
16 & 0.10 & 0.46 & 0.44 & 5523.431 & 5521.809 & 5521.696 \\
17 & 0.06 & 0.33 & $\mathbf{0 . 6 0}$ & 5406.431 & 5404.851 & $\mathbf{5 4 0 4 . 5 5 9}$ \\
18 & 0.45 & 0.48 & 0.08 & 4111.372 & 4111.316 & 4113.15 \\
20 & 0.07 & 0.27 & $\mathbf{0 . 6 5}$ & 4392.285 & 4391.146 & $\mathbf{4 3 9 0 . 5 4 4}$ \\
23 & 0.20 & 0.46 & 0.34 & 4118.352 & 4117.4 & 4117.23 \\
26 & 0.41 & 0.41 & 0.18 & 4729.668 & 4729.448 & 4729.813 \\
28 & 0.12 & 0.35 & $\mathbf{0 . 5 3}$ & 5373.769 & 5372.656 & $\mathbf{5 3 7 2 . 3 4 3}$ \\
29 & 0.46 & 0.37 & 0.18 & 5217.791 & 5217.718 & 5218.44 \\
30 & 0.08 & 0.32 & $\mathbf{0 . 6 0}$ & 5597.777 & 5596.301 & $\mathbf{5 5 9 5 . 6}$ \\
31 & $\mathbf{0 . 8 9}$ & 0.11 & 0.00 & $\mathbf{4 9 1 9 . 3 7 5}$ & 4921.154 & 4923.373 \\
33 & 0.00 & 0.04 & $\mathbf{0 . 9 6}$ & 4816.7 & 4813.931 & $\mathbf{4 8 1 1 . 2 8}$ \\
34 & 0.05 & 0.30 & $\mathbf{0 . 6 5}$ & 4651.213 & 4649.648 & $\mathbf{4 6 4 8 . 9 4 2}$ \\
35 & 0.09 & 0.28 & $\mathbf{0 . 6 3}$ & 5132.313 & 5131.1 & $\mathbf{5 1 3 0 . 4 4 7}$ \\
36 & 0.27 & 0.47 & 0.27 & 5104.89 & 5104.147 & 5104.327 \\
38 & 0.21 & $\mathbf{0 . 5 4}$ & 0.25 & 5451.796 & $\mathbf{5 4 5 1 . 0 7 6}$ & 5451.946 \\
\hline
\end{tabular}

Table B2

Non-Parametric SIC posterior probabilities and parametric MIC model LOOIC scores for each subject in Experiment 2. SIC results have been combined to match the architecture output of the MIC states. Winning models for each method have been bolded for clarity. For 19 of 27 subjects the two models provide converging evidence for the same processing architecture within that participant. For 8 subjects there is insufficient evidence to diagnose processing architecture reliably. 
Non-Parametric

\begin{tabular}{l|lll|lll}
\hline Subject & Positive & Serial & Negative & \multicolumn{1}{c}{$>\mathbf{0}$} & \multicolumn{1}{c}{$=\mathbf{0}$} & \multicolumn{1}{c}{$<$} \\
\hline 1 & 0.11 & $\mathbf{0 . 5 1}$ & 0.38 & 5351.268 & $\mathbf{5 3 5 0 . 2 0 8}$ & 5350.459 \\
2 & 0.00 & 0.11 & $\mathbf{0 . 8 9}$ & 4918.753 & 4912.984 & $\mathbf{4 9 0 6 . 6 4 5}$ \\
3 & 0.02 & 0.30 & $\mathbf{0 . 6 8}$ & 5759.499 & 5756.66 & $\mathbf{5 7 5 6 . 5 5}$ \\
4 & 0.00 & 0.42 & $\mathbf{0 . 5 8}$ & 4619.311 & 4615.015 & $\mathbf{4 6 1 2 . 6 8 2}$ \\
5 & 0.00 & 0.23 & $\mathbf{0 . 7 7}$ & 5447.426 & 5443.734 & $\mathbf{5 4 4 1 . 8 6 1}$ \\
6 & 0.00 & 0.09 & $\mathbf{0 . 9 1}$ & 5224.726 & 5220.768 & $\mathbf{5 2 1 7 . 6 6 2}$ \\
7 & 0.01 & 0.35 & $\mathbf{0 . 6 4}$ & 4990.462 & 4987.668 & $\mathbf{4 9 8 6 . 7 3 5}$ \\
8 & 0.01 & $\mathbf{0 . 5 7}$ & 0.43 & 5152.388 & $\mathbf{5 1 4 8 . 4 2 4}$ & 5149.943 \\
10 & 0.00 & 0.11 & $\mathbf{0 . 8 9}$ & 4048.983 & 4042.56 & $\mathbf{4 0 3 2 . 6 4 6}$ \\
12 & 0.01 & 0.48 & $\mathbf{0 . 5 0}$ & 3828.352 & 3824.988 & $\mathbf{3 8 2 4 . 3 7 2}$ \\
13 & 0.01 & $\mathbf{0 . 7 5}$ & 0.24 & 3711.696 & $\mathbf{3 7 0 7 . 5 3 9}$ & 3707.708 \\
14 & $\mathbf{0 . 6 1}$ & 0.35 & 0.04 & 4543.672 & 4544.191 & 4545.876 \\
15 & 0.00 & 0.44 & $\mathbf{0 . 5 6}$ & 4796.728 & 4792.847 & $\mathbf{4 7 9 1 . 7 3 3}$ \\
16 & 0.11 & $\mathbf{0 . 8 3}$ & 0.06 & 4613.86 & $\mathbf{4 6 1 1 . 8 0 2}$ & 4614.269 \\
17 & 0.00 & 0.30 & $\mathbf{0 . 7 0}$ & 5349.032 & 5344.418 & $\mathbf{5 3 4 3 . 4 1 2}$ \\
18 & 0.00 & 0.16 & $\mathbf{0 . 8 4}$ & 4233.509 & 4229.175 & $\mathbf{4 2 2 6 . 1 1 3}$ \\
19 & 0.30 & $\mathbf{0 . 6 9}$ & 0.01 & 5649.069 & $\mathbf{5 6 4 8 . 7 1 9}$ & 5651.798 \\
20 & 0.05 & $\mathbf{0 . 8 7}$ & 0.09 & 4627.707 & $\mathbf{4 6 2 4 . 6 4 5}$ & 4626.92 \\
22 & 0.00 & 0.36 & $\mathbf{0 . 6 4}$ & 5112.13 & 5107.24 & $\mathbf{5 1 0 4 . 0 9 7}$ \\
23 & 0.00 & 0.14 & $\mathbf{0 . 8 6}$ & 4736.31 & 4732.364 & $\mathbf{4 7 2 9 . 5 7 1}$ \\
24 & 0.01 & 0.13 & $\mathbf{0 . 8 7}$ & 5585.233 & 5582.555 & $\mathbf{5 5 8 0 . 7 7 4}$ \\
25 & 0.00 & 0.20 & $\mathbf{0 . 8 0}$ & 5176.875 & 5174.127 & $\mathbf{5 1 7 2 . 7 7 9}$ \\
26 & 0.02 & $\mathbf{0 . 6 7}$ & 0.31 & 4652.475 & $\mathbf{4 6 4 8 . 7 3 4}$ & 4650.03 \\
\hline
\end{tabular}

Table B3

Non-Parametric SIC posterior probabilities and parametric MIC model LOOIC scores for each subject in Experiment 3a. SIC results have been combined to match the architecture output of the MIC states. Winning models for each method have been bolded for clarity. For all subjects the two models provide converging evidence for the same processing architecture within that participant. 
Non-Parametric

\begin{tabular}{l|lll|lll}
\hline Subject & Positive & Serial & Negative & \multicolumn{1}{c}{$>\mathbf{0}$} & \multicolumn{1}{c}{$=\mathbf{0}$} & \multicolumn{1}{c}{$<0$} \\
\hline 102 & 0.21 & $\mathbf{0 . 7 7}$ & 0.02 & 4742.082 & $\mathbf{4 7 4 1 . 5 8 8}$ & 4744.986 \\
103 & 0.03 & 0.46 & $\mathbf{0 . 5 1}$ & 5057.422 & 5055.497 & $\mathbf{5 0 5 4 . 9}$ \\
104 & 0.01 & 0.23 & $\mathbf{0 . 7 6}$ & 4764.043 & 4761.209 & $\mathbf{4 7 5 9 . 6 8 1}$ \\
105 & 0.00 & 0.33 & $\mathbf{0 . 6 7}$ & 4365.818 & 4359.832 & $\mathbf{4 3 5 3 . 8 0 4}$ \\
106 & 0.25 & $\mathbf{0 . 7 3}$ & 0.02 & 5279.32 & $\mathbf{5 2 7 8 . 2 2 3}$ & 5281.763 \\
107 & 0.04 & 0.41 & $\mathbf{0 . 5 5}$ & 4987.729 & 4985.733 & $\mathbf{4 9 8 4 . 8 3 3}$ \\
108 & 0.00 & 0.16 & $\mathbf{0 . 8 3}$ & 5338.403 & 5335.459 & $\mathbf{5 3 3 3 . 5 4}$ \\
109 & 0.16 & $\mathbf{0 . 6 6}$ & 0.17 & 4124.312 & $\mathbf{4 1 2 2 . 8 9 7}$ & 4123.74 \\
110 & 0.00 & 0.33 & $\mathbf{0 . 6 7}$ & 4873.737 & 4869.166 & $\mathbf{4 8 6 6 . 5 8 2}$ \\
111 & 0.01 & 0.48 & $\mathbf{0 . 5 1}$ & 5184.38 & 5180.955 & $\mathbf{5 1 8 0 . 4 9 2}$ \\
112 & 0.28 & $\mathbf{0 . 6 7}$ & 0.05 & 4968.543 & $\mathbf{4 9 6 7 . 6 7 1}$ & 4969.61 \\
113 & $\mathbf{0 . 7 3}$ & 0.27 & 0.00 & $\mathbf{5 5 3 2 . 8 0 4}$ & 5534.003 & 5537.33 \\
114 & 0.00 & 0.15 & $\mathbf{0 . 8 5}$ & 5215.691 & 5212.633 & $\mathbf{5 2 1 1 . 6 7 9}$ \\
115 & 0.07 & $\mathbf{0 . 9 3}$ & 0.00 & 4637.575 & $\mathbf{4 6 3 5 . 0 8 5}$ & 4638.889 \\
116 & 0.00 & 0.27 & $\mathbf{0 . 7 2}$ & 4207.557 & 4203.212 & $\mathbf{4 2 0 0 . 8 2 5}$ \\
117 & 0.04 & $\mathbf{0 . 9 0}$ & 0.06 & 4261.915 & $\mathbf{4 2 5 9 . 2 7 5}$ & 4262.575 \\
120 & 0.00 & 0.29 & $\mathbf{0 . 7 1}$ & 5651.242 & 5647.655 & $\mathbf{5 6 4 6 . 0 7 2}$ \\
121 & 0.00 & 0.23 & $\mathbf{0 . 7 7}$ & 5461.453 & 5458.159 & $\mathbf{5 4 5 6 . 6 1 3}$ \\
122 & 0.22 & $\mathbf{0 . 7 7}$ & 0.01 & 4934.552 & $\mathbf{4 9 3 3 . 6 5 4}$ & 4937.014 \\
123 & 0.00 & 0.30 & $\mathbf{0 . 7 0}$ & 5013.595 & 5008.34 & $\mathbf{5 0 0 5 . 3 5 1}$ \\
124 & 0.02 & 0.28 & $\mathbf{0 . 7 0}$ & 5856.172 & 5853.788 & $\mathbf{5 8 5 3 . 6 2 7}$ \\
125 & 0.00 & 0.12 & $\mathbf{0 . 8 8}$ & 5020.41 & 5016.172 & $\mathbf{5 0 1 2 . 9 1 4}$ \\
126 & 0.05 & $\mathbf{0 . 6 2}$ & 0.33 & 5387.982 & $\mathbf{5 3 8 5 . 9 8 8}$ & 5386.532 \\
\hline Total & 1 & 8 & 14 & & & \\
\hline Ta16 B4 & & & & & &
\end{tabular}

Table B4

Non-Parametric SIC posterior probabilities and parametric MIC model LOOIC scores for each subject in Experiment 3b. SIC results have been combined to match the architecture output of the MIC states. Winning models for each method have been bolded for clarity. For all subjects the two models provide converging evidence for the same processing architecture within that participant. 